\title{
Fitting the spectral energy distributions of galaxies with CIGALE : Code Investigating GALaxy Emission
}

\author{
E. Giovannoli ${ }^{1}$ and V. Buat ${ }^{2}$ \\ ${ }^{1}$ Department of Physics, University of Western Cape, Private Bag X17, 7535, Belville, Cape \\ Town, South Africa \\ email: elodie.giovannoli@gmail.com \\ ${ }^{2}$ Laboratoire dAstrophysique de Marseille, OAMP, Université Aix-marseille, CNRS, 38 rue \\ Frédéric Joliot-Curie, 13388 Marseille, cedex 13, France \\ email: veronique.buat@oamp.fr
}

\begin{abstract}
We use the code CIGALE (Code Investigating Galaxies Emission: Burgarella et al. 2005; Noll et al. 2009) which provides physical information about galaxies by fitting their UV (ultraviolet)-to-IR (infrared) spectral energy distribuition (SED). CIGALE is based on the use of a UV-optical stellar SED plus a dust IR-emitting component. We study a sample of 136 Luminous Infrared Galaxies (LIRGs) at z 0.7 in the ECDF-S previously studied in Giovannoli et al. (2011). We focus on the way the empirical Dale \& Helou (2002) templates reproduce the observed SEDs of the LIRGs. Fig. 1 shows the total infrared luminosity $\left(\mathrm{L}_{I R}\right)$ provided by CIGALE using the 64 templates (x axis) and using 2 templates (y axis) representative of the whole sample. Despite the larger dispersion when only 1 or 2 Herschel data are available, the agreement between both values is good with $\Delta \log \mathrm{L}_{I R}=0.0013 \pm 0.045$ dex. We conclude that 2 IR SEDs can be used alone to determine the $\mathrm{L}_{I R}$ of LIRGs at $\mathrm{z} \sim 0.7$ in an SED-fitting procedure.
\end{abstract}

Keywords. galaxies: evolution — infrared: galaxies

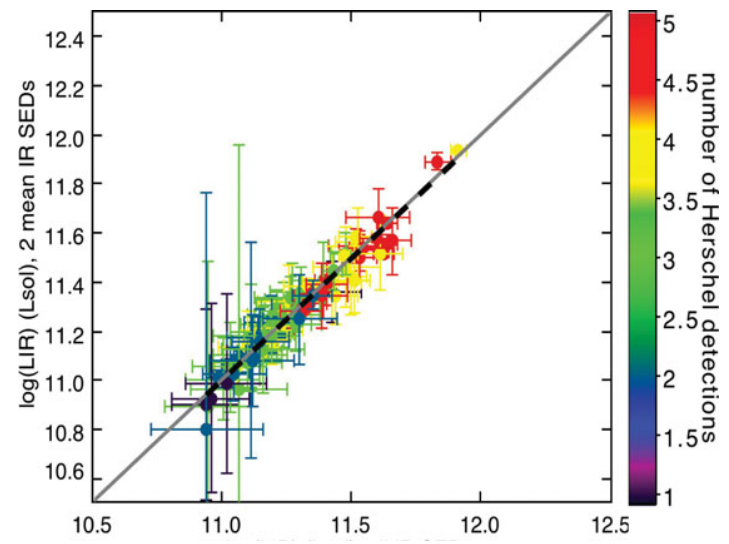

Figure 1. $\mathrm{L}_{I R}$ derived by CIGALE using the IR templates from Dale \& Helou (2002) library. On the $\mathrm{x}$ axis $\mathrm{L}_{I R}$ is derived using the 64 templates from the IR library and on the $\mathrm{y}$ axis $\mathrm{L}_{I R}$ is derived using only 2 IR templates representative of the whole sample of 136 LIRGs.

\section{References}

Burgarella, D., Buat, V., \& Iglesias-Páramo, J. 2005, MNRAS, 360, 1413

Dale, D. A. \& Helou, G. 2002, ApJ, 576, 159

Giovannoli, E., Buat, V., Noll, S., Burgarella, D., \& Magnelli, B. 2011, A\& A, 525, 150

Noll, S., et al. 2009, MNRAS, 507, 1793 